MATEC Web of Conferences 31, 03014 (2015)

DOI: $10.1051 /$ matecconf/ 20153103014

(C) Owned by the authors, published by EDP Sciences, 2015

\title{
The Motion Control of Profile Milling Flying Saw
}

\author{
Yuhua Zhang ${ }^{1,2, a}$, Xinhuan Song ${ }^{1,2}$ \\ ${ }^{1}$ Tianjin Key Laboratory of the Design and Intelligent Control of the Advanced Mechatronical System, \\ Tianjin University of Technology, Tianjin 300384, China \\ ${ }^{2}$ School of Mechanical Engineering, Tianjin University of Technology, Tianjin 300384, China
}

\begin{abstract}
Profile milling flying saw is one of the most advanced flying saws in the modern world, its tracking system driven by servo motor and its sawing system using profile milling method. This flying saw has many advantages, such as simple control system, less jamming signal, higher dimensional accuracy, flat section and convenient operation. Since the profile milling flying saw was launched, it has been put into a wide applications. The paper gives a brief introduction of the working principles of tracking system and makes a specific analysis of its motion. The movement patterns of saw cutting round pipes and square pipes are given in the sawing system and the mathematical models of saw cutting square pipes are established by analysis. In fact, the methods mentioned above have been used in practice. The dimensional accuracy of sawing welded pipe has reached the requirements of British BS standards and come up to the world advanced level.
\end{abstract}

Keywords: flying saw; tracking system; sawing system; dimensional accuracy

\section{Introduction}

Profile milling flying saw (known as flying saw for short) is important equipment that could be used in the welded pipe production line. After finishing the process of the formation of the welded pipe, the tracker of the flying saw tests the production speed of the welded pipe on-line. When the tracker and the welded pipes are at synchronized operation, the sawing device will imitate the shape of the welded pipe (round pipes or square pipes) to cut with a specified length [1]. So the motor system of the flying saw mainly consists of two parts: tracking system and sawing system. Tracking system achieves synchronous motion with welded pipe and control dimensional accuracy of sawing welded pipe. Sawing system does intelligent cutting according to the shape of the welded pipe, that's profile milling.

The flying saw control system is a kind of typical multi-axis control system and takes advantage of the MC206 controller which is based on Trio to control each phase of the movement of flying saw [2], especially in the realization of the $S$ curve movement and in the process of cutting for imitation shape cutting of square pipe. In MC206 controller, the technology of high performance 32 bit DSP of Trio is adopted, with the functions of four axis servo or stepping, in addition, there is an encode input shaft [3]. The controller software Motion Perfect has a total of eight shaft configurations and the application settings of four servos or stepper shaft can be realized in the controller, which can realize the control of flying saw vehicle, rotary table and two saw blades in flying saw.
The Table area is used to storage data generated by curve equations of motion and the CAMBOX command invokes the Table data to realize gradual control to each movement phase of flying saw machine, making it be able to move along the given motion curve.

\section{Tracking System}

\subsection{Working principle}

Fig. 1 shows the marching welded pipe lead the movement of velocity measuring roller, and let pulse generator send out signal Fp, which is corresponds to the speed of welded pipe, and then the pulse signal enters the proportion multiplier. According to the pulse signal the computer sends out variable speed control instruction in accordance with the scheduled program, making it form the speed pulse signal Fm after frequency division of Fp, The counter sends message to the computer after counted according to the computer and Fp control information. Then the computer will import new information to the counter, and import new variable speed control instruction to the multiplier, in order to control the speed of flying sawing machine. Fm transform to power pulse Fs through servo driver amplification, driving servo motor to turn the corresponding angle according to the input pulse. Servo motor decelerates through the gear $\mathrm{Z1} / \mathrm{Z2} / \mathrm{Z3}$ and $\mathrm{Z4}$ (sometimes it's triple deceleration) driving the flying saw vehicle through the gear and rack device. The flying saw vehicle's speed and displacement is related to the pulse

a Corresponding author: Yuhua Zhang, huan166@126.com

This is an Open Access article distributed under the terms of the Creative Commons Attribution License 4.0, which permits unrestricted use, distribution, and reproduction in any medium, provided the original work is properly cited. 
signal frequency and pulse number.

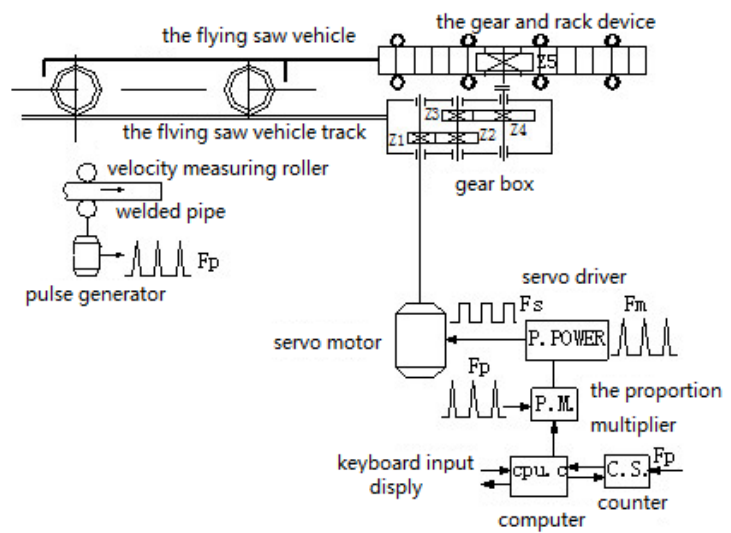

Fig.1 working principle schematic diagram of tracking system

\subsection{Motion analysis}

Fig.2 shows the movement of flying saw tracking system which can be divided into five periods: waiting period $\mathrm{AB}$, forward tracking period $\mathrm{BC}$, forward synchronous period $\mathrm{CE}$ (point $\mathrm{D}$ is the start time of flying saw vehicle after a certain period of stabilization while it enters into the synchronization period), forward deceleration period EF, reverse operation period FI, in which FG for reverse acceleration period, $\mathrm{GH}$ for reverse synchronization period, HI for reverse decelerate period.

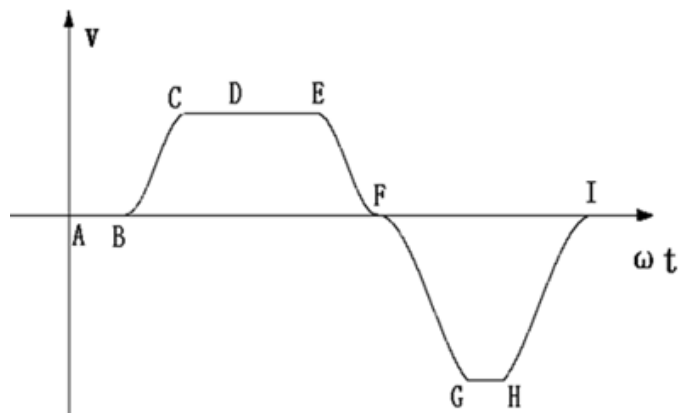

Fig.2 Motion sketch map of flying saw vehicle

The mathematical equations used in the process of tracking process will directly affect the dimensional accuracy and the power of the servo motor.

The mathematical equation which is used at present is:

Forward tracking period $\mathrm{BC}$ :

$$
\begin{gathered}
s=x m \frac{\Phi}{2 \pi \omega}\left[\frac{\pi}{\Phi} \omega t-\sin \left(\frac{\pi}{\Phi} \omega t\right)\right] \\
v=\frac{d s}{d t}=x m \bullet \sin ^{2}\left(\frac{\pi}{2 \Phi} \omega t\right) \\
a=\frac{d v}{d t}=x m \frac{\pi}{2 \Phi} \omega \bullet \sin \left(\frac{\pi}{\Phi} \omega t\right)
\end{gathered}
$$

Where $m$ is the pulse equivalent ( $\mathrm{mm} / \mathrm{pulse}$ ), $x$ is the peed pulse frequency of welded pipe (pulse/s), $\Phi$ is the traveling angle of gear which will drive flying saw vehicle during forward tracking period, $\omega$ is the angular velocity of gear which will drive flying saw vehicle $(\mathrm{rad} / \mathrm{s}), t$ is variable of time during tracking period (s), $t$ $=0 \sim \mathrm{T} 1, \mathrm{~T} 1$ is the tracking time

Forward deceleration period of EF:

$$
\begin{gathered}
s=x m \frac{\Phi}{2 \pi \omega}\left\{\frac{\pi}{\Phi} \omega t-\sin \left[\frac{\pi}{\Phi}(\Phi+\omega t)\right]\right\}+s_{1}+s_{2} \\
v=\frac{d s}{d t}=x m \bullet \sin ^{2}\left[\frac{\pi}{2 \Phi}(\Phi+\omega t)\right] \\
a=\frac{d v}{d t}=x m \frac{\pi}{2 \Phi} \omega \bullet \sin \left[\frac{\pi}{\Phi}(\Phi+\omega t)\right]
\end{gathered}
$$

Where $s_{2}$ is the trips of flying saw vehicle during synchronous period $(\mathrm{mm}), t$ is variable of time during forward deceleration period, $t=0 \sim \mathrm{T} 3$, is the time of forward deceleration.

Reverse acceleration period FG:

$$
\begin{gathered}
s=s_{1}+s_{2}+s_{3}-k x m \frac{\Phi}{2 \pi \omega}\left[\frac{\pi}{\Phi} \omega t-\sin \left(\frac{\pi}{\Phi} \omega t\right)\right] \\
v=\frac{d s}{d t}=-k x m \bullet \sin ^{2}\left(\frac{\pi}{2 \Phi} \omega t\right) \\
a=\frac{d v}{d t}=-k x m \frac{\pi}{2 \Phi} \omega \bullet \sin \left(\frac{\pi}{\Phi} \omega t\right)
\end{gathered}
$$

Where $s_{3}$ is the trip of flying saw vehicle during forward deceleration $(\mathrm{mm}), t$ is variable of during reverse acceleration period, $t=0 \sim \mathrm{T} 4, \mathrm{~T} 4$ is the time of reverse acceleration.

Reverse decelerate period HI:

$$
\begin{gathered}
s=s_{1}+s_{2}+s_{3}-s_{4}-s_{5}-k x m \frac{\Phi}{2 \pi \omega}\left\{\frac{\pi}{\Phi} \omega t-\sin \left[\frac{\pi}{\Phi}(\Phi+\omega t)\right]\right\} \\
v=\frac{d s}{d t}=-k x m \bullet \sin ^{2}\left[\frac{\pi}{2 \Phi}(\Phi+\omega t)\right] \\
a=\frac{d v}{d t}=-k x m \frac{\pi}{2 \Phi} \omega \bullet \sin \left[\frac{\pi}{\Phi}(\Phi+\omega t)\right]
\end{gathered}
$$

Where $s_{5}$ is the trip of flying saw vehicle during reverse synchronization period $(\mathrm{mm}), t$ is variable of time during reverse deceleration period, $\mathrm{t}=0 \sim \mathrm{T} 6, \mathrm{~T} 6$ is the time of reverse deceleration.

Where $k$ is:

$$
k=\frac{\sqrt{T_{1}\left(T_{1}+T_{2}\right)}}{T_{1}}
$$

Motion diagram made with computer programming according to results calculated with the above equation (see Fig.3). 


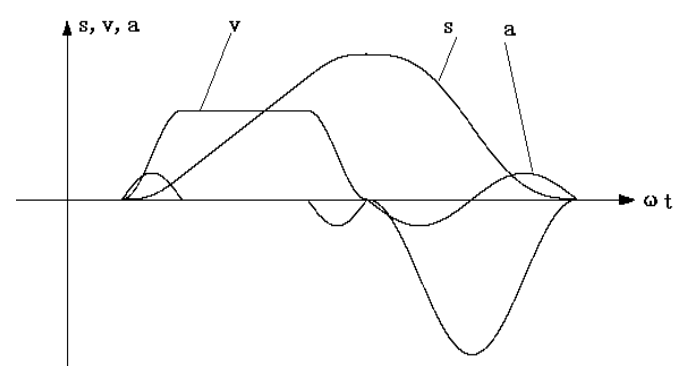

Fig.3 Flying saw vehicle's motion diagram

With the equations in practice, the flying saw will be neither rigid shock nor flexible shock. Therefore the flying saw device will come back faster without increasing the driving toque of the servo motor, resulting in increasing the sawing dimensional accuracy. The pulses during operation period and reverse operation period are exactly the same. At the end of a working cycle, the flying saw vehicle just stops at the null point and there is no error of the second run [4]. So the flying saw vehicle can achieve the control of speed and position.

\section{Sawing System}

\subsection{Motion analysis}

Under the polar coordinates, profile milling's movement can be divided into radial feed motion and rotation of the turntable (see Fig.4). The combination of radial feed motion and rotary mechanism rotation can make all kinds of cutting trajectory [5], resulting in the cutting action on round, square and rectangular pipes. The saw blade makes revolution along with the turntable; but at the same time, it also has its own rotation and radial motion [6].

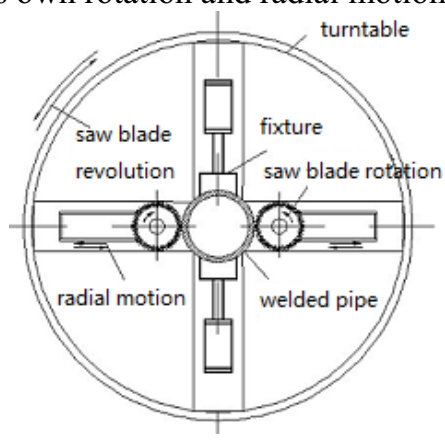

Fig.4 Profiling milling flying saw cutting structure

\subsection{Mathematical model}

In the radial motion, according to the differences of the shape of the cutting pipes, the radial movements are different [7]. When cutting round pipes (see Fig.5), saw blade radial feed fast at first, when closing to the pipe wall, radial feed slow to cut through the pipe, and then saw blade stop radial feeding and turntable start spinning, resulting in saw blade performing cutting though the walls of the pipes, rotating nearly 180 degrees and the pipe cut off.

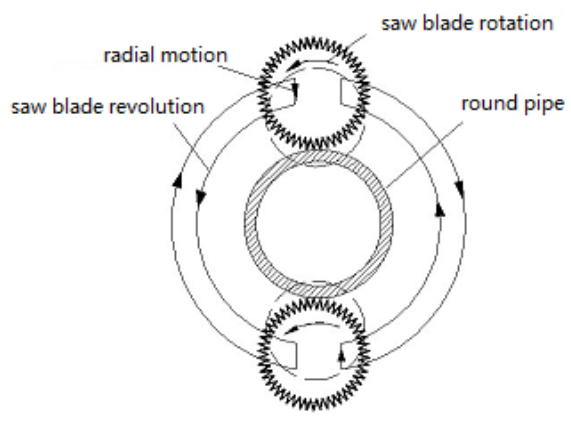

Fig.5 The movement of round pipes sawing

When cutting the square pipe (see Fig.6 (a)), the saw blade does radial motion with the turntable running. With the movement of the turntable, the distance of the saw blade which is installed at it to the square pipe wall is different, which requires the saw blade to keep doing radial motion while the turntable is moving. If the turntable cuts in from an angle of the square pipe, the feed rate should be the biggest when the turntable runs to the steel plane (see Fig.6 (b)), if the turntable keeps moving to the adjacent angle, the saw blade should do radial backward movement; if the turntable runs past the adjacent angle and keeps moving, the saw blade feeds again until it goes to the plane and then runs backward to cut the relative angle. Another blade also does the same movement. In this way, the turntable runs with a 180 degree and the square pipe is cut off. During the cut-off procedure, the saw blade has to do twice feed and retreat radial motions [8]. In order to finish this action, the control method of interpolation of the turntable servo axis and the feed servo axis is adopted. The two axes do interpolation movement through the MC206 motion-controlled instruction. Likewise, the turntable servo axis works as the principal axis and the feed servo axis as the secondary axis to do the radial interpolation motion along with the running of the turntable.

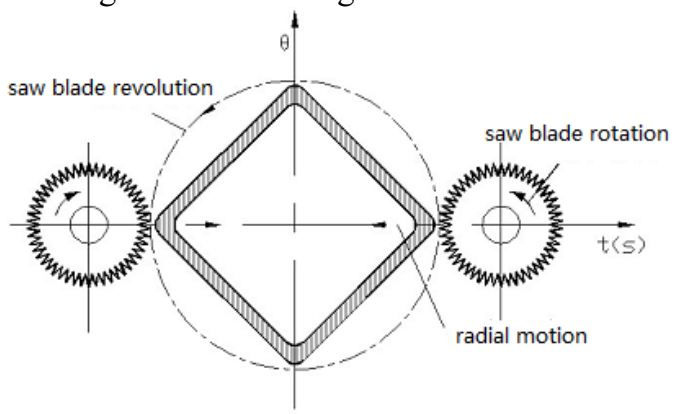

(a)

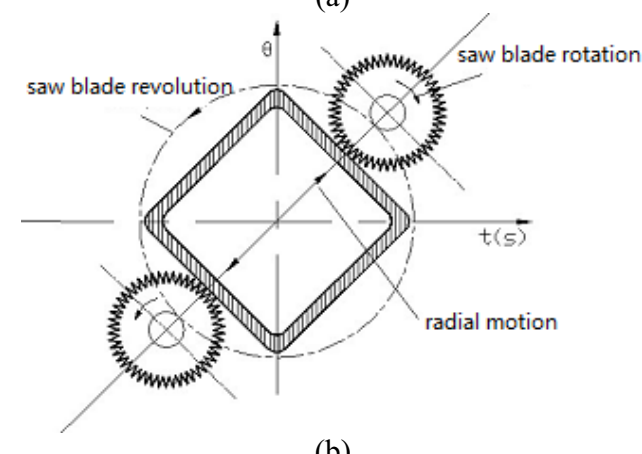

(b)

Fig.6 Cutting square pipes motion diagram 
MC206 can complete two axes interpolation motion through CAMBOX instructions. Calculate the number of saw blade displacement corresponds to per rotation angle of turntable, using the method of mathematical model, and then to save the corresponding values into the TABLE for the use of CAMBOX instruction.

The method of mathematical model and numerical calculation of TABLE are as follows:

Set $a$ for the length of $\mathrm{AB}$ of square pipe, $b$ for the length of BC (see Fig.7).

Then $\mathrm{OA}=\frac{\sqrt{a^{2}+b^{2}}}{2}$, saw blade radial displacement is $s$, $\theta$ is the rotation angle of turntable. With the movement of the turntable saw blade walking along the wall, divide 180 degrees which is the turntable's rotation into 900 shares, calculate each a radial displacement of the saw blades value into the TABLE. While execute CAMBOX command, turntable rotation one angle, the saw blade makes a corresponding radial motion.

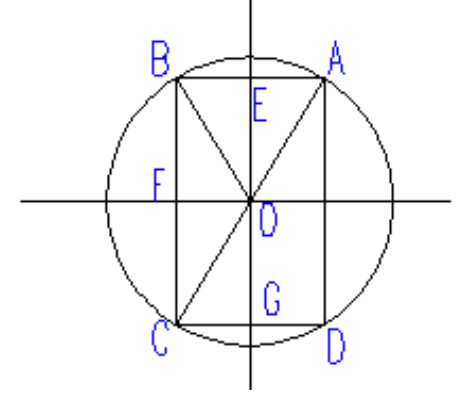

Fig.7 Radial feed movements

The movement of radial feed can be divided into four parts (see Fig.7).

- Saw blades move from A to E, both saw blades radial movement direction inward.

The radial displacement is:

$$
s_{1}=O A-\frac{b}{2 * \cos (\angle A O E-\theta)}
$$

- Saw blades move from E to B, both saw blades radial movement direction outward.

The radial displacement is:

$$
s_{2}=O A-\frac{b}{2 * \cos (\theta-\angle A O E)}
$$

- Saw blades move from B to F, both saw blades radial movement direction inward.

The radial displacement is:

$$
s_{3}=O A-\frac{a}{2 * \cos (\angle A O F-\theta)}
$$

- Saw blades move from $\mathrm{F}$ to $\mathrm{C}$, both saw blades radial movement direction outward.

The radial displacement is:

$$
s_{4}=O A-\frac{a}{2 * \cos (\theta-\angle A O F)}
$$

According to the mathematical model analysis, programming could be made for production.

\section{Conclusions}

The paper gives a motion analysis of tracking and sawing system of profile milling flying saw. The mathematical equations, compensation measures, the working principle diagram are all applied in practical production. Practice shows that after using these solutions, the dimensional accuracy of sawing welded pipe has reached the requirements of British $\mathrm{BS}$ standards and come up to the world advanced level, and can be completely competent the task of sawing welded pipe.

\section{References}

1. Guo Jifu, Zheng Hongyan, et al. Numeric control profiling saw cutting-off technology of REW welded pipe. Welded Pipe and Tube. 29(3): 33-35(2006)

2. Trio Motion Technology Ltd. Trio motion control cont. United Kingdom: Trio Motion Technology $\operatorname{Ltd}(2004)$

3. Cong Shuang, Li Zexiang. The practical technology of motion control. Publishing House of Electronics Industry(2012)

4. Chai Xiaoyan. The kinematical analysis of flying-saw following system. Heavy Machinery. (1):16-18(2008)

5. Luan Chengzhong. Profile milling saws study on optimal control of the cutting control method. Mechanical College of Tonqi University(2005)

6. Lin Jiankang. Saddle manufacture of pipe-cutting machine. Mechanical and Electrical Engineering Technology. 32(1):28-29(2003)

7. Zhang Yuhua, Li Yunlong. Kinematic analysis of flying saw vehicle. Journal of Tianjin University of Technology. 24(5): 22-26 (2008)

8. Su Zhinan. The motion control of profile milling flying saw based on controller MC206. The 2nd International Conference on Information Science and Engineering (ICISE2010) 